\title{
Optical tests on submarine and land vehicle periscopes in parallel with environmental tests
}

\author{
Michel Honlet ${ }^{1}$, Kay Wehrenberg ${ }^{1}$ \\ ${ }^{1}$ Airbus DS Optronics GmbH (Airbus Defence and Space), 73447 Oberkochen, Germany \\ michel.honlet@airbusDS-optronics.com, kay.wehrenberg@airbusDS-optronics.com
}

\begin{abstract}
Modern periscopes for submarines and armoured vehicles include components like daylight cameras, thermal cameras and laser range finders, often in addition to an optical channel for direct sight. Like any complex component which has been conceived for use in land and water vehicles, all periscopes must be subjected to tests under severe mechanical, thermal and climatic loading conditions. It is fundamentally important and safety relevant that the optical performance is maintained during such extreme conditions.

The special feature is that beyond these numerous environmental tests, several optical tests must be carried out additionally and simultaneously, which is quite challenging. For example, measuring the parallelism of multiple optical axes at different wavelengths is a difficult task, especially when measurements are achieved outside of test chambers and while the tests are running. Usually, hurdles like fog, condensation and the vibrations of the tested object must be overcome.

Although it is a difficult task, only if optical measurements are carried out in parallel with environmental simulation tests the long-term reliability and the performance of optical systems and periscopes can be guaranteed, despite environmental influences.
\end{abstract}

Key words: optical tests, environmental tests, cameras, periscopes, reliability.

\section{Introduction}

In addition to the traditional direct view channel by means of glass optics, modern periscopes include additional components such as a daytime camera, thermal imaging camera and laser rangefinders. Like all complex components designed for all-terrain vehicles, periscopes also run through a whole series of mechanical, thermal and climatic load tests.

However, a special feature is that not only the interactions between a periscope and its environment must be examined, but rather that optical tests must take place simultaneously. For example, to measure the alignment of several optical axes at different wavelengths requires elaborate examinations. Since these are carried out during simulation methods optical tests often run outside of climatic chambers.

This document will demonstrate how optical measurements are carried out in parallel with environmental simulation tests, in order to ensure the performance of optical systems at long range despite environmental influences.

\section{Resolution Measurement}

One of the essential parameters for the quality of optical systems is the accurate reproduction of an observed object. As a measure for resolving power when measuring an optical system, the object-side angle $\varepsilon$ is determined, that is, the angle yielded from a minimally resolvable line pair of width $a$ at a distance $f^{\prime}$ to an objective, ( see Fig. 1).

According to Foucault's method the resolving power $\varepsilon$ in [ $\mathrm{mrad}$ ] is

$\varepsilon=a / f^{\prime}$

In the ideal case, the resolving power $\varepsilon$ is equivalent to the value specified by the theory of diffraction.

For the evaluation a target plate with stepped, variable sized pattern is generally used ( see Fig. 2). The measurement goal is to determine the field of the Foucault test, the grid lines of which are only just separable when being observed visually in all directions, that is, in horizontal, vertical and diagonal orientation. 


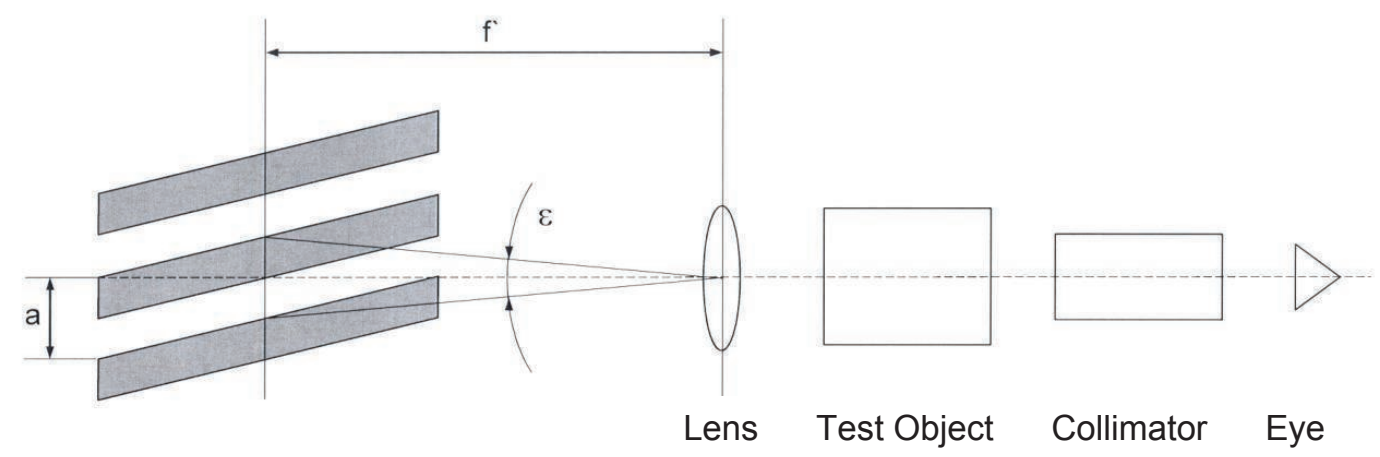

Fig. 1. Example of horizontal line pairs to determine $\varepsilon$

The measurements usually take place under ideal conditions, meaning statically and under standard environmental conditions. For optical targeting and observation devices used in allterrain vehicles, ships or aircraft, environmental disturbances such as temperature and mechanical oscillations are additionally active, which of course could also occur in combination.

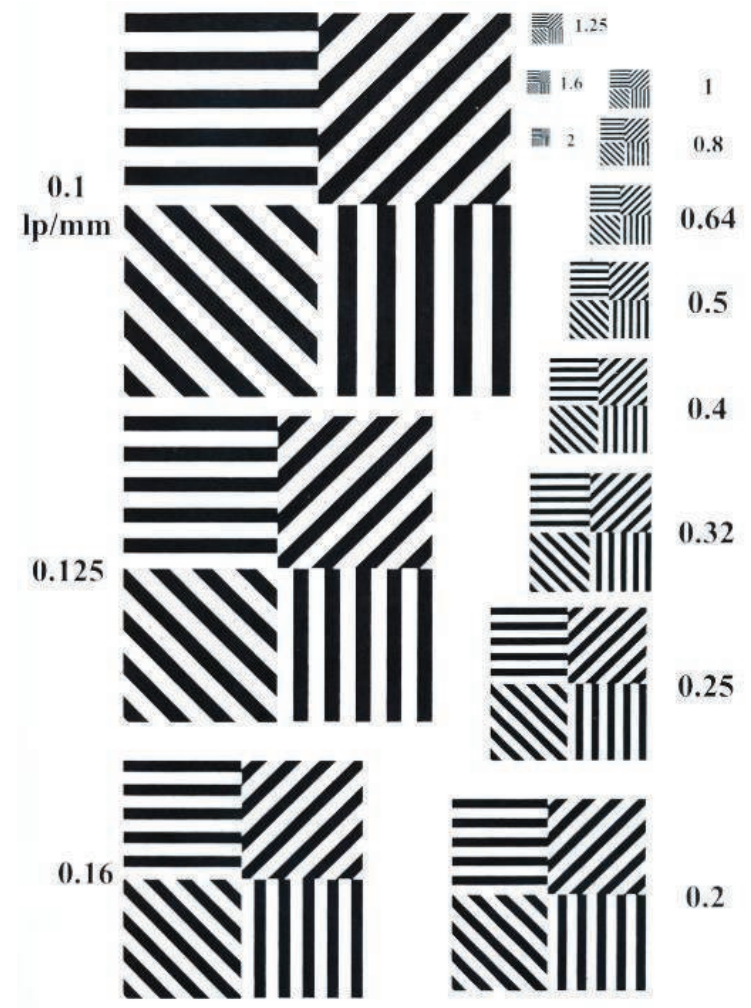

Fig. 2. Example of a target plate for a Foucault test.

For measurements under temperature, the climate zones of NATO Standardization Agreement (STANAG) 2895 are usually taken as the basis; that is, the ambient temperature of the devices to be tested is set at $-19{ }^{\circ} \mathrm{C},-32^{\circ} \mathrm{C}$ or $-46{ }^{\circ} \mathrm{C}$ for cold according to climate zones $\mathrm{C} 0, \mathrm{C} 1$ and $\mathrm{C} 2$. For tests in dry heat, climate zones A1, A2 or A3 must be applied, which corresponds to a maximum ambient temperature in the series $71^{\circ} \mathrm{C}, 63^{\circ} \mathrm{C}$ and $58^{\circ} \mathrm{C}$, respectively, under consideration of solar radiation. Due to linear thermal expansion of the adjacent construction of optical assemblies, the focal distance of the optical system also changes further, which inevitably influences image sharpness. In direct view (= purely optical image generation, meaning image is visible without an electronic sensor), this behaviour can easily be corrected by manually changing the eyepiece-side dioptre setting.

For optical sensors such as thermal imagers or charge-coupled device (CCD) cameras, compensation must be made for the thermal errors. This athermalisation can take place optically by correcting the focus position as a function of the ambient temperature or also as a mechanical compensation by means of an intelligent pairing of materials. However, further temperature-related aspects must still be considered for the sensors. The transmission values and the refractive indices of the materials suitable for this wavelength range change as a result of the temperature influence, in particular in the case of infrared optics.

In the case of CCD cameras it affects the sensitivity of the CCD chips. A measurement in the temperature range is therefore imperative for verification of the temperature influence on the image quality of the sensors.

To evaluate the resolution quality in direct view, an autocollimator telescope is used instead of a target plate, in which a target corresponding in principle to the target plate from figure 2 has been integrated. This collimator which serves to virtually shift the target plate to infinity can also be used for the measurement of CCD cameras.

For the measurements in the infrared band, an infrared collimator is used, so that the heated target becomes visible in the thermal image. As a consequence, by reproducing the images on a monitor, a geometric resolution can be carried out even with the sensors.

For the evaluation of image quality of the sensors, however, additional significant variables must be determined for which special collimators are used. 
For CCD cameras, this is the MTF value (Modulation Transfer Function), understood as the relationship between the contrast of details at the edges of an object and the contrast of same details represented in the image, so the contrast reduction of details. For thermal imaging devices, the MRTD value (Minimum Resolvable Temperature Difference) is considered being the significant variable for the resolving power, understood as the minimally resolvable temperature difference of an object to its ambient temperature.

Because the collimators needed for the measurements are not temperature-resistant or thermal influences would change the optical properties, they must be positioned outside of the test chamber and the chambers must be equipped with corresponding windows both for the visual and infrared wavelength range.

In order to prevent icing by condensate freezing over at low temperatures, the windows are designed as double-glazed windows. The individual panes are separated from one another here by a cold bridge and, in order to further prevent formation of condensation in the interior of the double window, the intermediate space is filled with nitrogen gas.

During the measurements, the influence of the windows on the measurement results must be taken into account, because - in particular in the near infrared range - considerable transmission losses occur. Figure 3 shows the typical setup within and outside of a temperature chamber when measuring the optical beam paths of a terrestrial periscope.

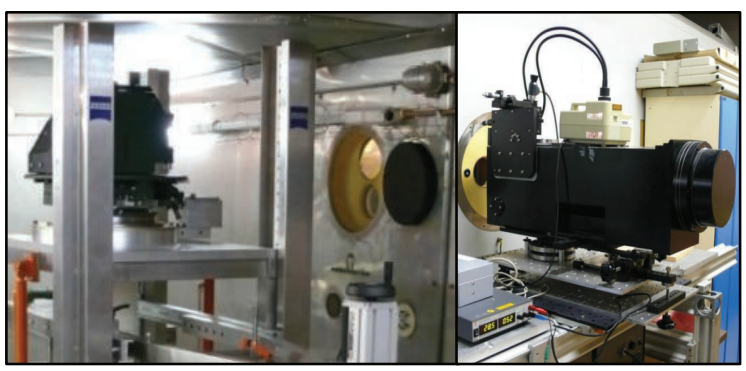

Fig. 3. Terrestrial periscope within and a collimator outside of the temperature chamber

The measurement of the optical variables takes place under vibrating conditions in the same manner as in the temperature test. All test spectrums specified for the respective devices are applicable in principle as load levels for the measurements. In contrast to temperature testing, in which the windows to be used are considered a source of error, potential sources of error must be observed under vibration which could be caused by relative movements of the test specimen with respect to the respective collimator. This applies to potential heading and pitch angles that could be caused in particular by large and/or heavy test specimens, but not to the translational amplitude of the shaker, because the observation takes place virtually in the infinite and thus no image offset is effective.

Potential angular movements caused by the test specimen can mostly be eliminated by considering the position of centre of gravity of the test specimen, including the test bracket, and by means of a multipoint control strategy.

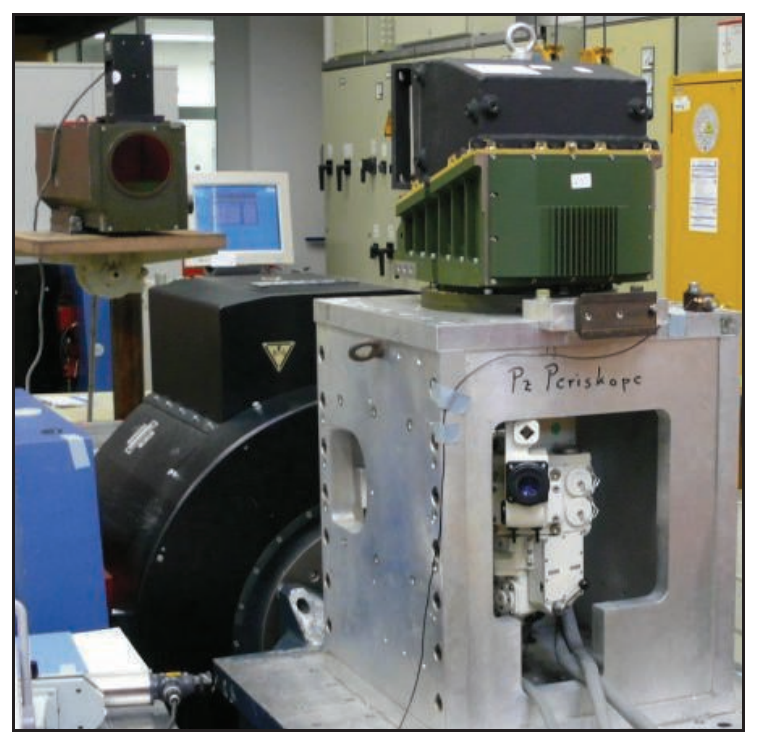

Fig. 4. Infrared collimator directed towards a terrestrial periscope mounted on a shaker

The test setup for measuring a periscope on the horizontal shaker is shown in Figure 4. The measured values that were determined under temperature and under vibration load ultimately serve as an important basis for calculating specific performance data, such as the maximum range for detecting or identifying objects.

\section{Jitter Measurement}

The evaluation of the resolution quality of optical sensors is made as described above, also under vibration. Traditionally a target is observed by the device to be examined and displayed on a monitor. The problem in this subjective evaluation is that during measurement the visual impression of a rendered target changes over time when using noise spectra. Factors like the individual visual acuity of the observer are added to this, as well as the fact that in the case of extensive measurement activity, the eyes tire over time, by which the measurement result may be influenced to a significant degree.

For an objective evaluation of the image oscillations caused by vibration, a jitter measurement is therefore conducted as an alternative or even additionally. The designation is somewhat 
misleading here because it deals not with the recording of image noise but rather with the actual image oscillations of the system to be evaluated.

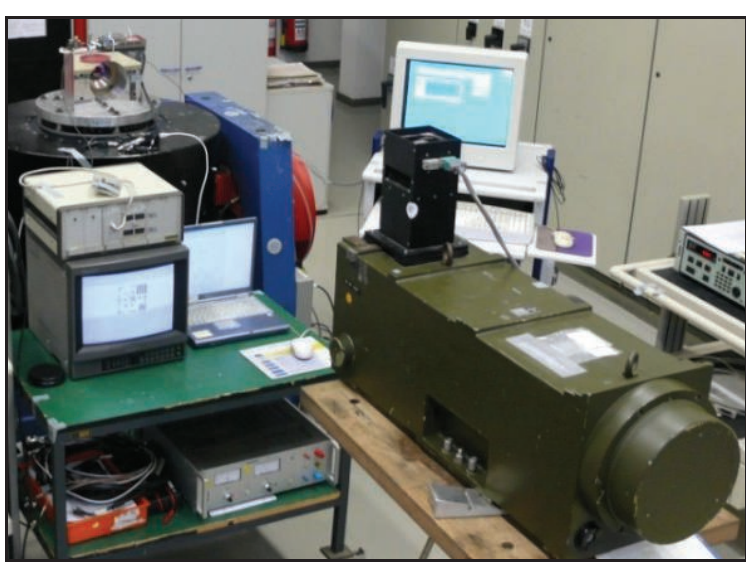

Fig. 5. Test setup for the jitter measurement on a thermal imaging device

The device to be evaluated is first aligned on the target of a collimator during the measurement and then a digital video sequence is recorded under vibration. A typical test setup for a jitter measurement on a vertical shaker is shown in Figure 5.

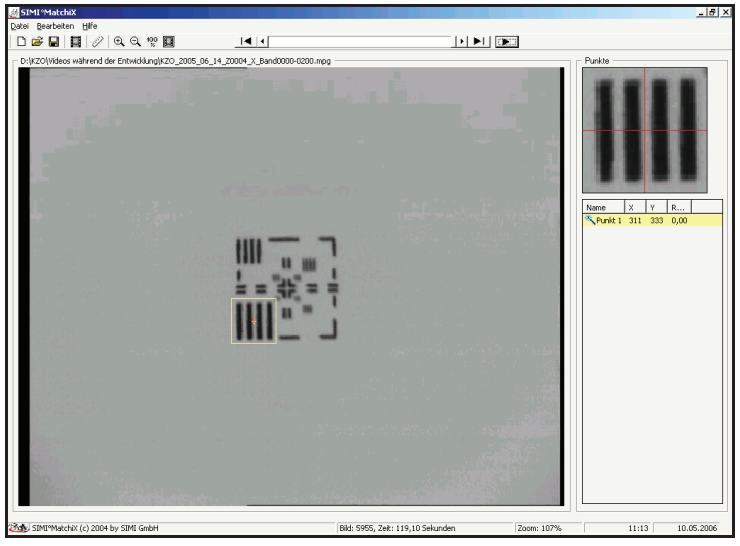

Fig. 6. Recording image oscillations with objecttracking software

The actual analysis subsequently takes place with the aid of an object-tracking software. Movement data is recorded by automatic tracking of a select image object ( see Fig. 6). The vibration-related offset with respect to the starting point is registered here frame by frame as $X$ and $Y$ coordinates. Moreover, the figure shows the target emitted by the infrared collimator and visualised by the thermal imaging device.

Usually an image sequence of over two minutes is recorded; in other words, at 50 frames per second, there are ultimately 6,000 frames available for the evaluation. The data pairs determined with the object-tracking program for the $X$ and $Y$ coordinates are exported into an
Excel program for calculation, by use of which the vectorial tray can be calculated as a rootmean-square (RMS) value. The calculated value in pixels can be converted directly into an angle known field of view and can therefore be specified, for example, as a quality feature for a production test, in which case a subjective test can be relinquished entirely.

A further application option of the method is in the optimisation of optical sensors. For example, an existing thermal imaging device had to be modified for a reconnaissance drone due to more stringent requirements placed on vibration-related image stability. For this, the actual condition was established with acceleration sensors by resonance measurements. Based on the measurement results, various weak spots were detected, which then had to be improved gradually.

The modification of the bearing structure and the zoom assembly may be cited in this case. The bearing structure of the thermal imaging device has been produced to date from a conventional aluminium alloy. Through the use of DISPAL $®$, a heavy-duty aluminium material with approx. $35 \%$ silicon and significantly higher strength and stiffness, a considerable improvement was able to be achieved.

Furthermore, during observation under vibration with a stroboscopic light, relative movements with respect to the adjacent construction were discovered in the zoom assembly, which have a direct oscillating effect on the images of the thermal imaging device. Here, by changing the design of the bracket, a reduction in image unsteadiness was also able to be achieved.

However, other measures revealed only slight progress and would have been hardly perceptible using the traditional method, that is, the visual image evaluation. Here jitter measurements provide an objective value and because even the finest nuances are detectable, it thus serves as a decision aid for the acceptability of a measure. The sum of all modifications led to a significant reduction in image oscillations.
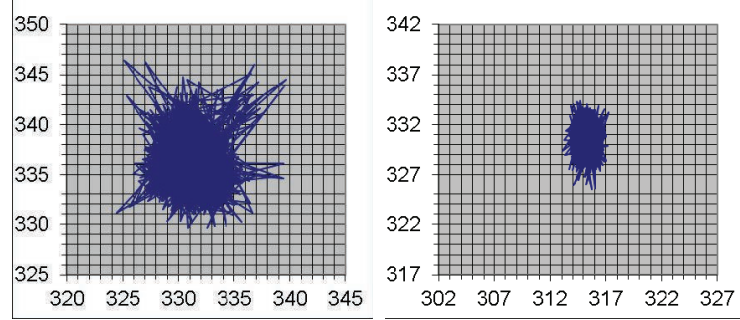

Fig. 7. Jitter measurement examples before and after modification 
The measured jitter value for the thermal imaging device amounted to 3.35 pixels (RMS)before modification and just 1.46 pixels (RMS) for the final version. Figure 7 shows the associated movement pattern of the measurements on a standardised scale; in other words, all 6,000 image points of the measurement have been combined graphically.

\section{Line of Sight (LOS) Stability}

A significant performance characteristic of optical targeting systems and devices is the line of sight stability, that is, the stability of the optical axes of all sensors, be it under temperature influence or even after mechanical loading. A collimator system is used for measurement, which usually comprises a visual alignment telescope for reference settings (zero position) and an automatic collimator telescope for the measurement of the visual beam path (CCD camera), as well as an infrared collimator for the infrared beam path of the thermal imaging device.

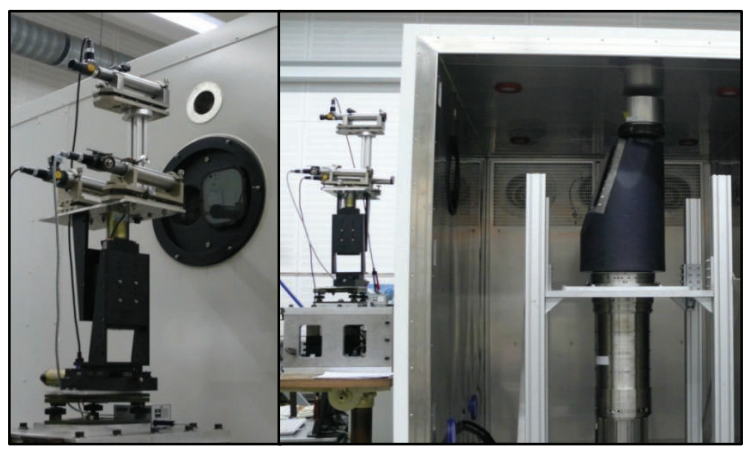

Fig. 8. Test setup to measure the line of sight stability of a maritime periscope

The collimators are connected to one another rigidly here and can be adjusted jointly with one another via a corresponding bracket. With the visual alignment telescope aligned on a mechanical reference on the test specimen itself or the test mount, the position of the collimator system with respect to the optical axis of the test specimen translationally in the $X$ and $Y$ position (focused on the reference mark), and rotationally horizontally and vertically (in automatic collimators), can be readjusted to the initial position at any time. This process is necessary, for example, in order to compensate for any potential movements of the test setup within the test chamber due to temperature loading.

Figure 8 shows a typical test setup for the measurement of a fully electronic periscope for submarines (OMS, Optronik Mast System), with the test specimen inside the test chamber and the collimator measuring system outside of it.
Diamond-cut surfaces in mirror quality on the test specimen or the test mount with a corresponding marking are used as a reference. The use of adhered mirrors is not permissible here, because adhesive bonds have their own properties and behaviour under temperature, which could considerably distort the measurement results. The measurement accuracy is about $15 \mu \mathrm{rad}$ or 3 arc seconds respectively.

Using known field-of-view variables over an optical path, the angle corresponding to a pixel in the monitor image is also known, whereby the angle of derivation can ultimately be calculated using vectors and with respect to the initial position. During the measurement, after setting the zero position of the collimators relative to the test specimen, the images of the thermal imaging device or the CCD camera are measured as follows:

a) Infrared beam path: In the infrared collimator, thermal radiation is emitted by means of a heated diffusion disc, which - limited by a diaphragm - is depicted by the thermal imaging device in the form of a small illuminated dot in the scene. A temperature-related change to the optical axis takes place by measuring the illuminated dot position change within the infrared image using a crosshairs generator; that is, the crosshairs generated on the monitor image can be shifted pixel by pixel to the current position of the illuminated dot.

b).Visual beam path: When measuring the visual beam path, the same process is used, but here the current position of the collimator mark is measured out by the automatic collimator telescope, instead of the illuminated dot.

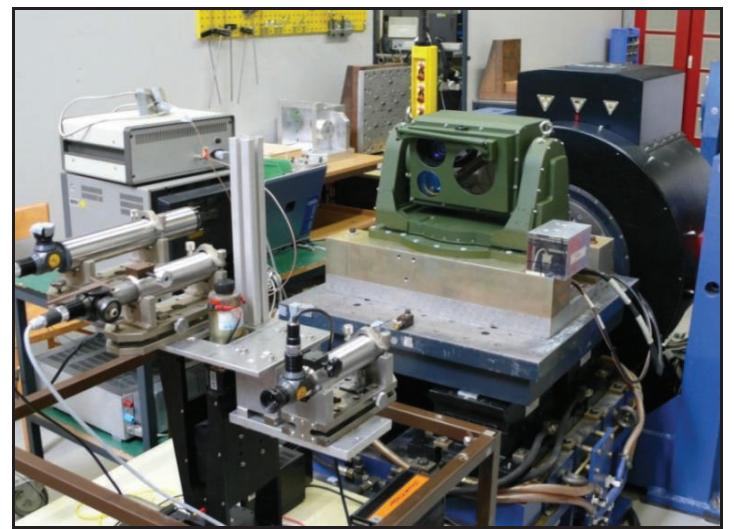

Fig. 9. Test setup for measuring the line of sight after vibration

When measuring the optical axes after mechanical loading, for example after vibration or a shock, the measurement takes place in the 
same way as the measurement under temperature.

In the case of directable targeting devices (devices that can be adjusted in elevation and azimuth) it is always necessary that after loading the device can be adjusted back to an electric zero position corresponding to the initial position before loading, provided the reference is not fixed on the device. The example in Figure 9 shows a device during the measurement following vibration.

\section{Optical Measurements on the Structure}

Under vibration load all technical systems have a tendency to develop certain inherent behaviour. Particularly in case of resonance, this leads to relative movements between the individual assemblies, which in the case of land vehicle periscopes respectively their optical assemblies results in image oscillations and thus to detriments in resolution quality.

For an optimisation of such systems, a traditional measurement of the image transfer behaviour with accelerometers is often inadequate, because decisive features like occurring angular movements are not recorded due to the translational direction of measurement. Using triaxial acceleration sensors, potential angular movements can indeed be determined, but this requires complex mathematic analysis systems that are capable of taking into account spatially occurring displacements, frequencies and phases, particularly in the case of random excitation.

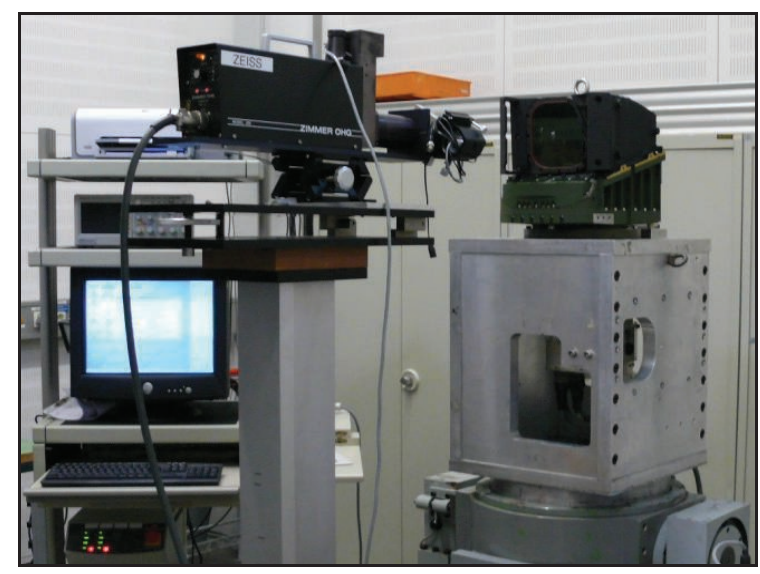

Fig. 10. Measurement of the image stabilisation quality on the motion simulator

The use of an electro-optical displacement sensor (optronic high-speed camera) offers a significantly easier procedure, by which angular movements can be recorded directly with the use of an automatic collimator adapter. The camera is aligned vertically to a monochrome edge (linear displacement measurement) or a mirror face (angular measurement) for the measurement. Without contact the camera converts the occurring linear or angular movements into proportional voltages, in one or even two axes.

During angular measurement, the measured signal can be rendered online by an evaluation device, for example an oscilloscope, and converted directly into the real angle. The gathered data thus provide the designer or mechanical engineer with a quick and important tool as the basis for required system modifications, if necessary also in conjunction with the results from corresponding acceleration measurements.

A further application option of the camera is to verify the line of sight stability of gyro-stabilised land vehicle periscopes with respect to specified tolerances. The basic requirement here is that the line of sight remains on a targeted point regardless of any vehicle movement during the "Target Lock" operating mode.

This verification can be carried out on a motion simulator capable of simulating rough terrain, by which the vehicle movements in terms of elevation and azimuth can be simulated at the same time ( see Fig. 10). Here, the full optical beam path is recorded by means of an auxiliary mirror on the eyepiece; that is, the result thus corresponds to the user's visual impression.

\section{References}

All figures and information shown here are from in-house. Additional sources were not used. 